\title{
Nanoscale
}

Check for updates

Cite this: Nanoscale, 2018, 10, 758

\section{Commensurability between protein nanotubes in contractile ejection nanomachines}

\author{
Sergey B. Rochal, (D) *a Daria S. Roshal, ${ }^{a}$ Anna E. Myasnikova ${ }^{a}$ and \\ Vladimir L. Lorman ${ }^{\text {b }}$
}

Contractile ejection nanomachines being sheath-tube assemblies create an opening in the cell membrane to translocate molecules or ions across it. Here, on the most structurally investigated examples of the bacteriophage T4 tail and pyocin R2, we show that the rearrangement of the sheath structure resulting in its contraction and twist occurs in such a way that the contracted sheath becomes commensurate with the inner tube. This fact dictates the previously unknown simple geometrical relationship between the nanotube symmetries. Using the Frank and van der Merwe classical theory of commensurability, we study an interaction between two protein nanotubes forming such nanomachines and obtain an expression for the corresponding energy, which depends on the tube structures and their mutual arrangement. The appearance of commensurability between the contracted sheath and the inner tube decreases both the interaction energy and the total energy of the system. It improves the nanomachine efficiency, since the energy gain obtained increases the torque of the inner tube piercing the cell membrane.

Received 17th September 2017 Accepted 3rd December 2017

DOI: $10.1039 / c 7 n r 06940 e$

rsc.li/nanoscale their high killing capacity, ${ }^{11}$ R-type pyocins and bacteriophages have attracted attention for antimicrobial and bioengineering applications. ${ }^{12-14}$

A significant breakthrough in the research of various biological nanomachines was induced by the methods of cryoelectron microscopy and X-ray crystallography, ${ }^{1,2,15}$ which in turn increased the scientific interest in these objects. Among the contractile ejection systems, the nanodevice of bacteriophage T4 is the most investigated. This bacteriophage attacking enterobacteria consists of a capsid containing viral DNA and a tail $^{1}$ formed by a pair of coaxial cylinders of equal length. Both tail tubes are composed of $M=23$ rings with 6 proteins per ring. Before intrusion into the host cell, the proteins of the outer envelope (so-called sheath) form loosely twisted spirals, and the tail has a so-called extended structure. Upon contact with the bacterium, the sheath changes its shape dramatically. Protein spirals are twisted more strongly and compressed. As a result, the outer shell decreases greatly in length, somewhat widens and turns into a more compact contracted structure. This leads to the piercing of the cell wall by the relatively rigid inner tail tube, which creates a pathway for the penetration of the viral DNA into the interior of the host cell. ${ }^{1}$ Despite the different functions, the structure of the bacteriophage $\mathrm{T} 4$ tail practically coincides with that of pyocin R2. ${ }^{6}$ Two nanoassemblies differ only in the number of rings (for pyocin $M=24$ ) and have some variations in geometry (including the length and twist of protein spirals).

According to the recent structural data on the pyocin R2 assembly ${ }^{6}$ and the bacteriophage $\mathrm{T} 4$ tail $^{1}{ }^{1}$ in both nano- 
machines the inner tube is commensurate with the extended outer one: the distance between the nearest rings and the turn angle between them are practically the same. This fact enforces us to raise a question whether the sheath contraction is a transition to another commensurate state of the sheath-tube system. The work of the syringe-like nanomachines is mainly produced at the expense of the internal energy stored in the extended sheath. ${ }^{1,6}$ However, the transition into the commensurate state would allow some increase of this work due to the additional decrease of the total energy of the system.

Modulated and commensurate systems are quite common in condensed matter physics. ${ }^{16}$ For example, such crystalline structures can be formed due to emerging sublattices ${ }^{17}$ or charge density waves,${ }^{18}$ by the periodic distortion of one initial lattice,${ }^{19}$ in magnetic systems. ${ }^{20}$ As we have recently shown, ${ }^{21}$ some commensurability is also possible in protein nanoassemblies, in particular, it occurs between two walls of the doubleshelled spherical capsids of Reoviridae and Cystoviridae families and between concentric spherical sublattices forming the icosahedral capsids of some marine viruses, in which the capsid includes protruding knob-like proteins.

In this paper, using and generalizing the classical theory of commensurability, which is typical of modulated crystal structures ${ }^{16}$ we show that in both the discussed nanomachines the commensurability between the outer and inner tubes reappears after the sheath contraction. Namely, the protein spirals of the outer tube contract and twist in such a way that they align along the larger diagonals of elementary cells forming the protein structure of the inner tube. Such structural organization of the contracted sheath reduces the energy of the interaction between the coaxial nanotubes and allows the increase of the torque created by the nanomachine.

Also, this paper studies an additional rational commensurability that is possible between the nanotubes of finite length. As we show here, if the distance between the nearest rings forming the inner tube is greater than the analogous one characterizing the outer tube, then some gain in the interaction energy can be achieved due to the appearance of the rational commensurability. It happens if the total length of the outer tube becomes equal to the half-integer number of distances between the rings in the inner tube. As we show here, this relationship exists between the total length of the contracted sheath and the distance between the rings in the inner tube of the bacteriophage T4 tail. However, the energy gain from this geometrical relationship is small, and in pyocin $\mathrm{R} 2$ there is no analogous rational commensurability.

\section{Results and discussion}

To begin with, below we study the commensurability and its features in the sheath-tube assembly of bacteriophage T4. As was already mentioned, both inner and outer tubes of this system are formed by ring-like hexamers consisting of $N=6$ proteins. The contraction of the bacteriophage sheath starts from the baseplate and is realized by the successive flattening of neighboring hexamers. In addition, the turn angle $\delta \varphi$ between the nearest hexamers changes and the sheath part, which is not yet contracted, rotates with respect to the inner tube as a whole. Thus, a front, which divides the initial and contracted sheath states, propagates along the tail. ${ }^{1,6,22}$ Note also that in the equilibrium contracted state the adjacent rings are turned by the angle $\delta \varphi=32.9^{\circ}$ and are spaced from each other by the distance $\delta z=16.4 \AA^{2} .^{2}$ Analogous parameters characterizing the structure of the inner tube, ${ }^{1} \delta z_{\text {in }}=40.2 \AA$ and $\delta \varphi_{\text {in }}=17.9^{\circ}$, practically coincide with those of the extended sheath, $\delta z_{\mathrm{ex}}=\delta z_{\mathrm{in}}, \delta \varphi_{\mathrm{ex}}=\delta \varphi_{\mathrm{in}}$, and the latter sheath structure is commensurate with the inner tube.

Let us introduce a cylindrical coordinate system with the rotation angle $\varphi$ and the $z$ axis directed along the bacteriophage tail. Then the protein positions can be specified by two indices $(n, m)$, where $n=0,1,2, \ldots \ldots, N-1 ; m=0,1,2, \ldots, M-1$; $M$ is the number of rings in the tube. In the contracted sheath tube the coordinates of the protein with indices $(n, m)$ in a cylindrical coordinate system are

$$
\begin{gathered}
\varphi_{n, m}=\varphi_{0}+n \frac{2 \pi}{N}+m \delta \varphi ; \\
z_{n, m}=z_{0}+m \delta z,
\end{gathered}
$$

where $\left(z_{0}, \varphi_{0}\right)$ is the shift of the protein $(0,0)$ with respect to the origin of the coordinate system. Note that positions (1) lie on helical lines (spirals), the number of which is equal to 6 $(N=6)$, and each of the lines turns on $\delta \varphi$ at the shift $\delta z$ along the $z$ direction. For the extended sheath tube and the inner one, the coordinates of the proteins can be determined similarly to (1), by shifting the coordinate origin and replacing the parameters $\delta \varphi$ and $\delta z$ with $\delta \varphi_{\text {ex }}$ and $\delta z_{\text {ex }}$, or with $\delta \varphi_{\text {in }}$ and $\delta z_{\text {in }}$, respectively. It is convenient to represent the tubular structures under consideration as cylinders decorated with parallelograms containing protein positions. Large parallelograms bounded in Fig. $1 \mathrm{~b}$ and $\mathrm{d}$ with solid black lines are the elementary cells of the inner tube. Smaller in size, colored parallelograms represent the protein locations in the structures of the extended (b) and contracted (d) outer tube. For the sake of simplicity we assume that the sheath protein positions coincide with the centers of smaller parallelograms. Fig. 1a and c (modified from ref. 1 and 23) demonstrate the sheath spiral in the extended and contracted states, respectively. The parameters of the sheath spirals shown in Fig. 1a $1 \mathrm{~b}$ are the same. In contrast, for the spirals shown in Fig. 1c and d these parameters are equal only approximately, since in the idealized contracted sheath (d) the protein helixes are located exactly along the longer diagonals of the elementary cells covering the inner tube surface. Due to this fact the angular shift $\delta \varphi$ between the adjacent hexamers in the idealized contracted sheath satisfies the following relationship:

$$
\delta \varphi=\frac{\delta z}{\delta z_{\text {in }}}\left(\delta \varphi_{\text {in }}+\frac{2 \pi}{6}\right)
$$

The proposed way to rationalize the contracted sheath arrangement is extremely accurate. The calculation of the $\delta \varphi$ 


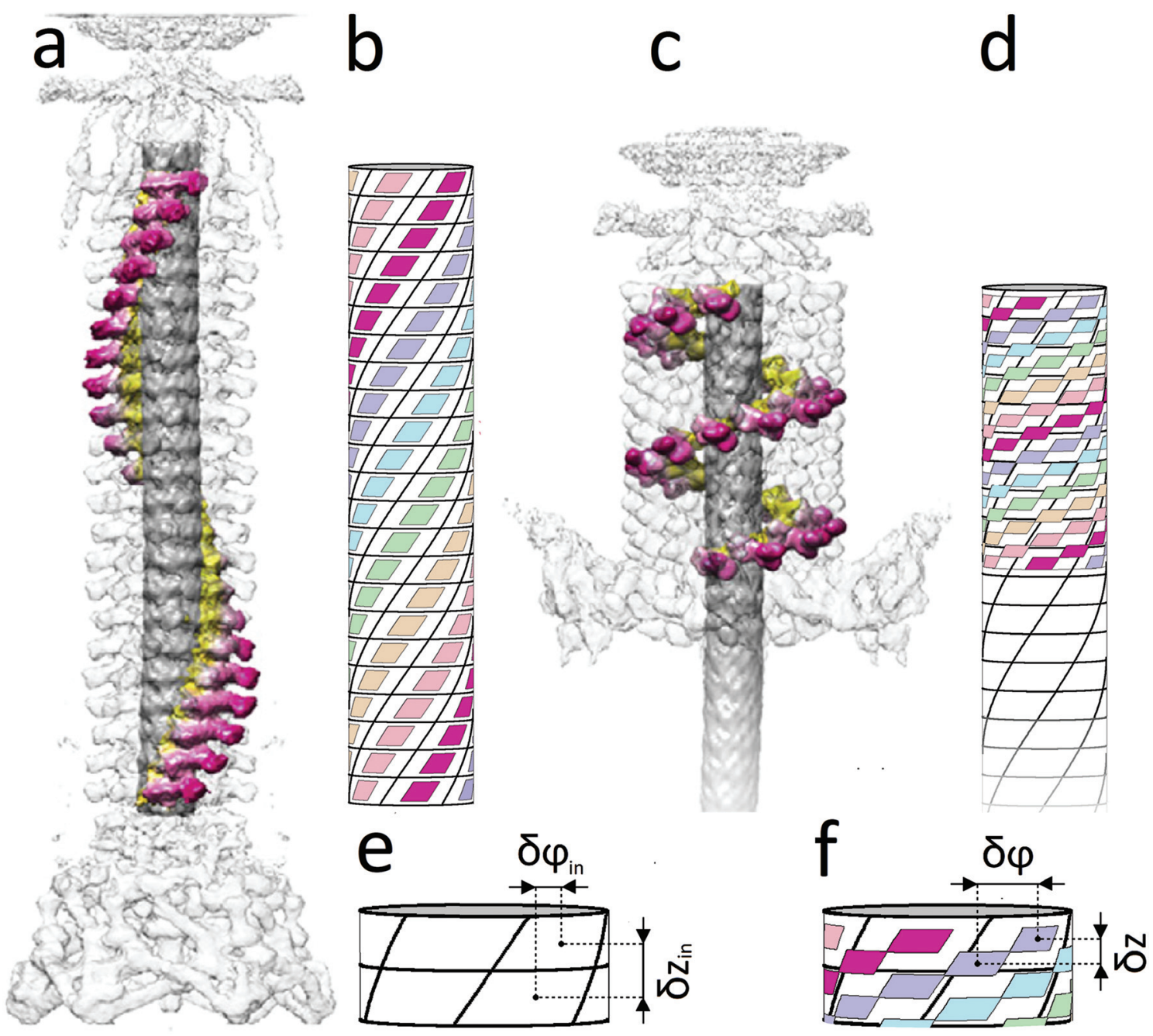

Fig. 1 Extended $(a, b)$ and contracted (c, d) states of the bacteriophage T4 tail. The panels $(a, c)$ (modified from ref. 23, where these figures were constructed using the data from ref. 1) show the protein spiral in the extended and contracted states, accordingly. The elementary cells of the inner tube (see panels $b, d$ ) are shown by cylindrical parallelograms bounded with solid black lines. Each cell corresponds to one protein molecule of the inner tube. The protein helixes of the sheath are represented by small parallelograms whose centers coincide with the protein positions (see panels b, d). The sides of these parallelograms are parallel to the boundaries of the elementary cells drawn on the inner tube surface. In the extended state $(a, b)$, the structures of the inner and outer tubes are commensurate and equivalent to each other: the centers of small and large parallelograms coincide. In the idealized contracted state (d), the protein helixes of the sheath are arranged along the diagonals of the inner tube elementary cells, and the length of the contracted sheath is exactly equal to the height of 9.5 cells drawn on the inner tube. Thin black circles show the upper and lower boundaries of the idealized sheath. The panels $(\mathrm{e}, \mathrm{f})$ demonstrate the definition of $\left(\delta z_{\text {in }}, \delta \varphi_{\text {in }}\right)$ and $\left(\delta z_{,} \delta \varphi\right)$ parameters for the inner tube and the contracted sheath, respectively. The parameters $\left(\delta z_{\mathrm{ex}}, \delta \varphi_{\mathrm{ex}}\right)$ of the extended sheath coincide ${ }^{1}$ with those of the internal tube.

value according to eqn (2) yields $32.2^{\circ}$, while the structural work $^{2}$ reports $\delta \varphi=32.9^{\circ}$ and, as far as we know, this latter $\delta \varphi$ value has not been revised in the subsequent studies.

The previously unknown but very simple commensurability relation (2) is not a single one for the bacteriophage T4 tail. According to the structural data for the outer ${ }^{2}$ and inner ${ }^{1}$ tubes, the total length of the contracted sheath $23 \delta z$ turns out to be equal, with good accuracy, to the 9.5 times the distance between the neighboring rings in the inner tube. Indeed, the calculation of $\delta z$ with the formula $9.5 \delta z_{\text {in }} / 23\left(\delta z_{\text {in }}=40.2 \AA\right)$ yields $\delta z=16.6 \AA$ instead of the actual value of $16.4 \AA^{2}{ }^{2}$ This coincidence, in our opinion, is not accidental. It shows that an additional rational commensurability along the $z$-direction between the contracted sheath and the inner tube exists. This commensurability relationship is taken into account in idealized Fig. 1d and will be deduced below.

In order to understand the structural relationships between the sheath and the inner tube, we will develop further and apply the widely known model ${ }^{17}$ proposed by Frank and van der Merwe. In its initial form the model explains the commensurability appearance in a simple one-dimensional system. The system is a linear chain of particles located in an external periodic field. The particle energy in this field is $W \cos \frac{2 \pi}{b} x$, where $W$ and $b$ are the field's amplitude and period, respectively, and $x$ stands for the particle coordinate. The neighboring 
particles of the chain are connected by springs and the model Hamiltonian is:

$$
H=\sum_{n} \frac{k}{2}\left(x_{n+1}-x_{n}-a_{0}\right)^{2}+W \cos \frac{2 \pi}{b} x_{n}
$$

where $a_{0}$ is the equilibrium distance between the particles, $k$ stands for a rigidity coefficient, $n$ is the particle number, and $x_{n}$ is the coordinate of the $n$-th particle. The first term of the Hamiltonian is responsible for the elastic interaction of the nearest neighbors. The second one takes into account the interaction with the external periodic field.

The minimization of (3) leads to a one-dimensional sineGordon equation that yields the equilibrium particle positions. ${ }^{24}$ When the parameters of the system, for example the equilibrium distance between particles $a_{0}$ or period $b$, change, a sequence of phase transitions, called the devil's staircase, arises. ${ }^{16,17}$ The deepest minima of the Hamiltonian (3) correspond to the cases where the equilibrium distance between the nearest neighbors is an integer multiple of the distance between the minima of the periodic potential.

In the frames of the model $^{17}$ one can also introduce a rational commensurability. Let us assume that all distances between the particles are equal to $a_{0}$ (it occurs provided $k \gg$ $W / b^{2}$ ) and, consequently, the particle coordinates are $x_{n}=a_{0} n+$ $\Delta$, where $\Delta$ is a constant. In this case only the second term in the energy (3) is non-zero and some specific relationships, which minimize the interaction of the chain fragment with the external field, exist. We refer to these relationships between $b, a_{0}$ and $\Delta$ as those of rational commensurability. Fig. 2 shows an example of this commensurability between the external harmonic field and the chain fragment (8 particles located at the centers of 8 elementary cells). The shown chain fragment is 4.5 times longer than the period $b$ and corresponds to 5 negative and 4 positive half-waves of the external field. It is easy to verify that the fragment's length and location are the most energetically favorable with respect to the periodic external field.

Note, however, that in the contracted state a gap of about $10 \AA$ appears between the inner and outer nanotubes, and unlike the extended state, the direct contact between the nanotubes is absent. When modeling the sheath contraction, this fact is often used in order to neglect or to downplay the role of

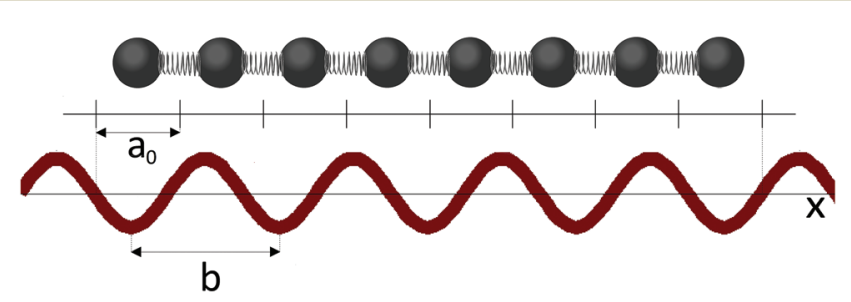

Fig. 2 Rational commensurability between the chain fragment and the external harmonic field. The chain fragment with the length equal to 4.5 periods of the external field is demonstrated. Since the fragment's length and location (with respect to the field plot) correspond to 5 negative half-waves and 4 positive half-waves of the field, the shown geometry is energetically favorable. the sheath-tube interaction. For example, the authors of a recent report in ref. 25 do not consider this interaction at all during the construction of their model and argue at the end of the article that it can only increase the energy of the extended state. In contrast, we state that this interaction is much more important since both the inner and outer nanotubes are periodic structures formed from large structural units with the size of the same order as the gap between nanotubes. Due to the large dipole moments of such structural units ${ }^{6}$ they interact with each other at distances greater than the gap length. However, it is difficult to construct a microscopic approach explicitly taking into account the above-described electrostatic interaction between nanotubes. So, below we will generalize the theory $^{17}$ and introduce a phenomenological potential determined by the structure of the inner rigid tube and model its influence upon the outer tunable sheath.

It is natural to assume that the minima of the desired interaction potential, reflecting the symmetry of the inner tube, coincide with the protein positions in the extended sheath, since the latter one is commensurate with the internal tubular structure. For the sake of simplicity, let us construct such simplest potential as a superposition of only two waves determined on the cylindrical surface.

As the first of them, it is reasonable to choose a planar wave with a period along the $z$ direction equal to the distance between neighboring rings in the inner tube: $V_{1}=-V_{1}^{0} \cos \left(k_{1} z\right)$, where $k_{1}=\frac{2 \pi}{\delta z_{\text {in }}}$ is the wave vector of this wave. To construct the second wave, let us recall that the protein positions of the inner tube like the positions (1) can be considered as located on a system of helical lines. Note also that there is an ambiguity in the choice of such a system since it is impossible to distinguish whether the neighboring hexamers are mutually turned by the angle $\delta \varphi_{\text {in }}$ or by angles $\delta \varphi_{\text {in }}+2 \pi q / N$ provided $q$ is an integer and $N=6$. So, one can use different systems of helical lines. The wave, whose troughs on the cylindrical surface form such a system with index $q$, is written as $V_{2}=$ $-V_{2}{ }^{0} \cos \left(k_{1} z+N \varphi\right)$, where $k_{2}=\frac{1}{\delta z_{\text {in }}}\left(\delta \varphi_{\text {in }}+q \frac{2 \pi}{N}\right)$. Let us stress that for any $q$ value the superposition of waves $V_{1}$ and $V_{2}$ has equivalent minima at the centers of the elementary cells drawn on the inner tube surface (see Fig. $1 \mathrm{~b}$ and d), while it has maxima at the cells' vertices. The horizontal boundaries of the cells are given by the troughs of the wave $V_{1}$. Different $q$ values correspond to different choices of the elementary cells. In particular, the ordinary cell tessellation shown in Fig. $1 \mathrm{~b}$ and $\mathrm{d}$ is determined by $q=0$. However, since the centers of elementary cells composing the contracted sheath are aligned along helical lines with $q=1$ (see Fig. 1d), below we will use the wave with this $q$ value to construct the desired phenomenological potential.

The consideration of the sheath contraction as a process and the discussion of the soliton-like ${ }^{6,22}$ boundary motion between the contracted and extended states of the outer tube are out of scope of this paper. Moreover, it should be emphasized that the nanomachine accomplishes work due to its 
internal energy, which is mainly stored in the extended sheath and is also not considered here. However, if the contracted sheath is characterized by such values of $\delta \varphi$ and $\delta z$, which decrease the interaction energy between the inner tube and contracted sheath, an additional resulting energy gain can also be used when the nanomachine is activated. So, below we study only the energy $E_{\text {int }}$ of the tube interaction, which is analogous to the second term in eqn (3):

$$
E_{\mathrm{int}}=-\sum_{n=0}^{N-1} \sum_{m=0}^{M-1}\left(V_{1}{ }^{0} \cos \left(k_{1} z_{m}\right)+V_{2}{ }^{0} \cos \left(k_{2} z_{m}+N \varphi_{m, n}\right)\right) \text {. }
$$

Since in the extended sheath state all the minima of the energy (4) simply coincide with the positions of sheath proteins, $E_{\text {int }}$ in this state has the global minimum value $E_{\text {int }}=$ $-N M\left(V_{1}{ }^{0}+V_{2}{ }^{0}\right)$. To calculate $E_{\text {int }}$ for the contracted sheath, we substitute the protein coordinates (1) into the energy (4). Then the latter one becomes a function of the four variables $\varphi_{0}, z_{0}$, $\delta \varphi$, and $\delta z$ with respect to which it should be minimized. Let us note that after the substitution of $\varphi_{0}=z_{0} k_{2}$ and eqn (2) into eqn (4), the second term in (4) takes its lowest possible value $-V_{2}{ }^{0} N M$. It is the energy gain due to the appearance of the commensurability (2). It occurs since the substituted equalities mean that all the protein positions are located in the troughs of the corresponding potential wave. Thus, eqn (4) becoming a function of two variables $z_{0}$ and $\delta z$ acquires the following form:

$$
E_{\mathrm{int}}=-V_{1}{ }^{0} \sum_{n=0}^{N-1} \sum_{m=0}^{M-1}\left(\cos \left(k_{1}\left(z_{0}+m \delta z\right)\right)\right)-V_{2}{ }^{0} N M .
$$

The above substitution does not fix the values of $z_{0}$ and $\delta z$, which are the only variables determining the contribution of the first term (proportional to $V_{1}{ }^{0}$ ) into energies (4) and (5). Therefore, an additional energetically favorable relationship between $\delta z, z_{0}$ and $\delta \mathrm{z}_{\text {in }}$ can be found by the minimization of eqn (5). Using the Euler formula $\mathrm{e}^{i \phi}=\cos \phi+i \sin \phi$ and summarizing the resulting geometric progression (its denominator is $\mathrm{e}^{i k_{1} \delta z}$ ) we obtain:

$$
E_{\text {int }}=-N V_{1}{ }^{0}\left(\operatorname{Re}\left(\frac{\mathrm{e}^{i 2 \pi \beta_{0}}\left(\mathrm{e}^{i 2 \pi \beta M}-1\right)}{\mathrm{e}^{i 2 \pi \beta}-1}\right)\right)-V_{2}{ }^{0} N M,
$$

where $\beta_{0}=\frac{z_{0}}{\delta z_{\text {in }}}, \quad \beta=\delta z / \delta z_{\text {in }}$.

Note that the energy (6) could hypothetically take a globally minimal value if the sheath does not shorten during the nanomachine action, but elongates in such a way that the product $k_{1} \delta z$ would be a multiple of $2 \pi$. In this case in the global minima of (6) the variables $\beta_{0}$ and $\beta$ would be integers. However, in reality, the sheath contracts and such simple integer commensurability between the inner and outer tubes is not realized in the systems under consideration. So, below we investigate the local minima of the interaction energy (6), where the system can find itself if the more complex rational commensurability appears. For this purpose we rewrite the real part of the complex fraction in eqn (6) as:

$$
\operatorname{Re}\left(\mathrm{e}^{i 2 \pi \beta_{0}} \operatorname{abs}(\Theta) \mathrm{e}^{i \gamma}\right),
$$

where $\Theta=\frac{\mathrm{e}^{i 2 \pi \beta M}-1}{\mathrm{e}^{i 2 \pi \beta}-1}$. Then, in the maxima of eqn (7), which correspond to the minima of eqn (6), $\mathrm{e}^{i 2 \pi \beta_{0}} \mathrm{e}^{i \gamma}=1$ and eqn (7) is reduced to

$$
\operatorname{abs}(\Theta)=\left|\frac{\sin \pi \beta M}{\sin \pi \beta}\right|
$$

The minima of the function (8) with respect to the variable $\beta$ are located at points $\beta=j / M$, where $j=1,2, \ldots, M-1$. Fig. 3 shows an example of such a function with $M=8$. This value is equal to the number of particles which form the chain fragment shown in Fig. 2. The local maxima of eqn (8) lie in the midpoints between the local minima with high accuracy. Therefore in the local minima of the energy (6):

$$
\begin{gathered}
\delta z \approx \frac{\delta z_{\text {in }}\left(m_{1}+\frac{1}{2}\right)}{M}, \\
z_{0} \approx \delta z_{\text {in }}\left(m_{2}-\frac{1}{4}\right)+\frac{\delta z}{2},
\end{gathered}
$$

where $m_{1}$ and $m_{2}$ are integers. Eqn (9) are also the conditions of the rational commensurability between an M-cell fragment of the one-dimensional periodic chain and a periodic potential with period $\delta z_{\text {in }}$ (see Fig. 2). In both cases the expression for $z_{0}$ reflects the fact that the fragment should be located symmetrically with respect to the oscillations of the external field.

Let us stress that in the above part of the paper we have minimized not the total energy of the system, but only its part corresponding to the interaction between the nanotubes forming the nanomachines under consideration. As is well

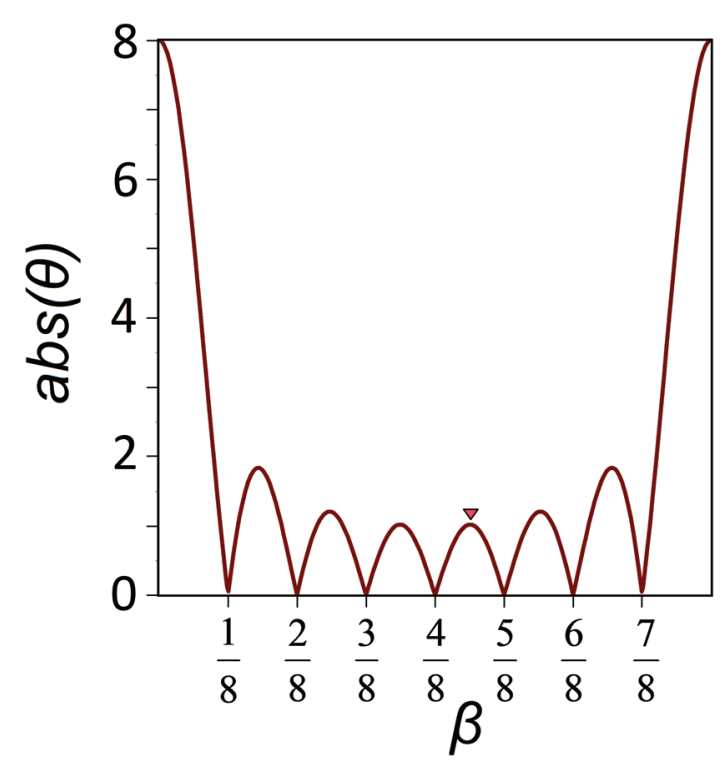

Fig. 3 The dependence of $\operatorname{abs}(\theta)$ on the $\beta$ value. This function is used for the rigorous analysis of the rational commensurability between coaxial nanotubes. The function with $M=8$ is shown. Its minima are located at the points $1 / 8,2 / 8, \ldots, 7 / 8$. The red triangle marks the maximum ( $\beta \approx 4.5 / 8$ ) corresponding to the rationally commensurate state shown in Fig. 2. 
known, only the minimization of total free energy yields the equilibrium parameters of any system. The fact that in the bacteriophage case the minima of both energies practically coincide (with respect to variables $\delta \varphi, \delta z$ ) is very surprising and it can be explained only as the natural way to increase the nanomachine efficiency.

It should be noted that the appearance of the rational commensurability reduces the tube interaction energy by a value, which is proportional to $N V_{1}{ }^{0}$. At the same time, if the condition (2) is satisfied (and, consequently, the protein positions in the sheath are located along the larger diagonals of the inner tube elementary cells), then the gain in the interaction energy due to the commensurability (2) is $V_{2}{ }^{0} N M$, which is $M$ times larger (provided $V_{1}{ }^{0} \approx V_{2}{ }^{0}$ ). Therefore, the rational commensurability is not very important for the considered systems.

Note also that since the condition (2) means that all positions of the contracted sheath are located in the troughs of one of the waves forming the tubes' interaction potential, the condition (2) describes the ordinary multiple commensurability, which is widespread in classical objects studied in condensed matter physics. One can also say that it is the simplest commensurability since we used the wave with $q=1$ and obtained the minimal rotation $2 \pi / 6$ in eqn (2). In a more general case, the minimal angle $2 \pi / 6$ in eqn (2) should be multiplied by an appropriate integer $q$.

Exactly the same commensurability (2) is manifested between the inner tube and the contracted sheath in pyocin R2. The structure of this contractile ejection nanomachine was recently studied in detail in ref. 6. Just like the tail of the bacteriophage $\mathrm{T} 4$, the syringe mechanism of pyocin R2 consists of two protein tubes; each of them, like in the bacteriophage case, is formed by six spirals. However, the number of rings in each of the tubes is 24 instead of 23 . The tubes are commensurate in the precontracted state, where their common parameters $^{6}$ are $\delta z_{\text {in }} \approx \delta z_{\text {ex }} \approx 38.4 \AA$ and $\delta \varphi_{\text {in }} \approx \delta \varphi_{\text {ex }} \approx 18.3^{\circ}$. The parameters $^{6}$ of the contracted sheath are $\delta z=16.2 \AA$ and $\delta \varphi=$ $33.1^{\circ}$. It is not difficult to verify that the protein spirals forming the structure of the contacted sheath are located approximately along the diagonals of the elementary cells composing the inner tube as it occurs in the bacteriophage T4. This fact indicates the commensurability existence. The usage of formula (2) yields an 'ideal' turn angle $\delta \varphi=33.03^{\circ}$ for the contracted sheath, while the measured ${ }^{6}$ value is $33.1^{\circ}$. As distinct from commensurability (2), the rational commensurability between the contracted sheath and the inner tube being not very energetically profitable is absent in this system: $24 \delta z / \delta z_{\text {in }} \approx 10.2$.

In addition it should be noted that 10 years ago the sheath of the $\varphi \mathrm{KZ}$ bacteriophage tail was investigated ${ }^{10}$ with comparatively low resolution and the symmetry of the internal tube was not determined. However, if in this bacteriophage, like in the both above-discussed systems, the extended sheath is commensurate with the internal tube, then, using the parameter values, ${ }^{10} \delta z_{\mathrm{ex}} \approx 34.1 \AA$, $\delta \varphi_{\mathrm{ex}} \approx 22^{\circ}$, and $\delta z \approx 17.9 \AA$, one can apply eqn (2). For the contracted sheath it yields the turn angle $\delta \varphi \approx 37.8^{\circ}$ instead of the reported ${ }^{10}$ one $36.7^{\circ}$. Thus, the struc- tural study ${ }^{10}$ also confirms the theory suggested since the small angular deviation obtained is very likely associated with the insufficient study resolution.

\section{Conclusions}

In this paper, using the structural data on the bacteriophage T4 tail $^{1,2}$ and pyocin R2 assembly, ${ }^{6}$ we investigated the commensurability between nanotubes forming syringe-like nanomachines and the commensurability influence on the nanomachine efficiency. The considered and similar nanodevices include two coaxial protein tubes, the outer one of which is tunable and can be in extended and contracted states. In both considered systems, as has been recently found, ${ }^{1,6}$ the inner tube is completely commensurate with the outer extended one. Here we show that after the sheath contraction a novel multiple commensurability arises between the nanotubes. The twisting of the sheath during its reconstruction occurs in such a way that the protein spirals forming the contracted sheath align along the diagonals of the inner tube elementary cells. In order to explain this finding, we developed the classic theory by Frank and van der Merwe ${ }^{17}$ and proposed a simple phenomenological potential, which describes the influence of the inner rigid tube upon the outer tunable one. Assuming that in the contracted sheath the neighboring protein rings are turned by the identical angle $\delta \varphi$ and separated by the same distance $\delta z$, we minimized the interaction energy obtained and deduced the relationship between the $\delta \varphi$ and $\delta z$ variables and the analogous parameters characterizing the rigid inner tube. This simple relationship is satisfactory with a very good accuracy in both the considered systems.

The extended state of the sheath is converted into the contracted one at the contraction front moving from the baseplate. ${ }^{1,2,22}$ At this boundary, the novel appearing commensurability minimizes the energy of the interaction between the inner tube and the contracted part of the outer one. So, at the contraction front the transformed and turned hexamer ring of the sheath falls into the minimum of the potential created by the inner tube that increases the torque between the tubes and, consequently, can be biologically expedient.

Along with the multiple commensurability, a less energetically profitable so-called rational commensurability can exist between the nanotubes of finite length. As we have shown, if the ring spacing $\delta z_{\text {in }}$ corresponding to the inner tube is greater than the analogous parameter $\delta z$ of the sheath, then the rationally commensurate situation can be energetically favorable and it is realized provided that the total length of the outer tube $M \delta z$ is equal to the half-integer number of $\delta z_{\text {in }}$ values. In the tail of bacteriophage $\mathrm{T} 4$ this relationship (with the coefficient 9.5) exists, however, in pyocin $\mathrm{R} 2$ it is absent.

On the one hand, the results obtained in this work show the need for subsequent high resolution structural studies of syringe-like nanosystems and, on the other hand, our findings are important to understand and model the nanomachine action. With the exception of two systems considered in this 
paper, the geometric parameters of the other analogous nanodevices have not been sufficiently studied. The determination of these parameters for the type VI secretion system, ${ }^{4,5}$ the metamorphosis-associated contractile arrays ${ }^{26}$ and other similar objects would make it possible to understand better the general patterns that allow nature to increase the efficiency of contractile ejection nanomachines. In addition, our generalization of the theory ${ }^{17}$ for the tubular case could be developed further. As is known, solitons naturally appear in the classical version of this theory. Therefore, we believe that in the subsequent work it will be possible not only to consider the commensurability between the coaxial nanotubes in contractile ejection nanomachines, but also to construct a more adequate model of their action, which will describe the soliton-like motion of the boundary between the extended and contracted states of the outer tube.

\section{Author contributions}

V. L. proposed an idea that in both states of the bacteriophage T4 tail the protein nanotubes forming the tail are commensurate. After V.L.'s sudden death S. R. found the explicit form of this commensurability. He also wrote the greater part of the text. D. R. made the computations and drew the figures. A. M. participated in the discussion of the results and text writing.

\section{Conflicts of interest}

There are no conflicts of interest to declare.

\section{Acknowledgements}

The authors are grateful to Peter Leiman, who proposed to consider the example of pyocin $\mathrm{R} 2$ in this paper. S. R. and D. R. gratefully acknowledge the financial support of the Russian Science Foundation, grant number 15-12-10004.

\section{Notes and references}

1 N. M. Taylor, N. S. Prokhorov, R. C. Guerrero-Ferreira, M. M. Shneider, C. Browning, K. N. Goldie, H. Stahlberg and P. G. Leiman, Nature, 2016, 533, 346-352.

2 P. G. Leiman, P. R. Chipman, V. A. Kostyuchenko, V. V. Mesyanzhinov and M. G. Rossmann, Cell, 2004, 118, 419-429.

3 V. A. Kostyuchenko, P. R. Chipman, P. G. Leiman, F. Arisaka, V. V. Mesyanzhinov and M. G. Rossmann, Nat. Struct. Mol. Biol., 2005, 12, 810.
4 M. Basler, M. Pilhofer, G. P. Henderson, G. J. Jensen and J. J. Mekalanos, Nature, 2012, 483, 182-186.

5 C. T. French, I. J. Toesca, T. H. Wu, T. Teslaa, S. M. Beaty, W. Wong, M. Liu, I. Schröder, P. Y. Chiou, M. A. Teitell and J. F. Miller, Proc. Natl. Acad. Sci. U. S. A., 2011, 108, 1209512100.

6 P. Ge, D. Scholl, P. G. Leiman, X. Yu, J. F. Miller and Z. H. Zhou, Nat. Struct. Mol. Biol., 2015, 22, 377.

7 G. Bönemann, A. Pietrosiuk and A. Mogk, Mol. Microbiol., 2010, 76, 815-821.

8 A. B. Russell, S. B. Peterson and J. D. Mougous, Nat. Rev. Microbiol., 2014, 12, 137.

9 Y. Michel-Briand and C. Baysse, Biochimie, 2002, 84, 499510.

10 A. Fokine, A. J. Battisti, V. D. Bowman, A. V. Efimov, L. P. Kurochkina, P. R. Chipman, V. V. Mesyanzhinov and M. G. Rossmann, Structure, 2007, 15, 1099-1104.

11 M. Kageyama, K. Ikeda and F. Egami, J. Biochem, 1964, 55, 59-64.

12 S. R. Williams, D. Gebhart, D. W. Martin and D. Scholl, Appl. Environ. Microbiol., 2008, 74, 3868-3876.

13 D. Scholl, M. Cooley, S. R. Williams, D. Gebhart, D. Martin, A. Bates and R. Mandrell, Antimicrob. Agents Chemother., 2009, 53, 3074-3080.

14 J. M. Ritchie, J. L. Greenwich, B. M. Davis, R. T. Bronson, D. Gebhart, S. R. Williams, D. Martin, D. Scholl and M. K. Waldor, Antimicrob. Agents Chemother., 2011, 55, 5469-5474.

15 S. Kanamaru, P. G. Leiman, V. A. Kostyuchenko, P. R. Chipman, V. V. Mesyanzhinov, F. Arisaka and M. G. Rossmann, Nature, 2002, 415, 553-557.

16 P. Bak, Rep. Prog. Phys., 1982, 45, 587.

17 F. C. Frank and J. H. Van der Merwe, Proc. R. Soc. London, Ser. A, 1949, 198, 216.

18 J. A. Wilson, F. J. Di Salvo and S. Mahajan, Adv. Phys., 1975, 24, 117-201.

19 R. Pynn, Nature, 1979, 281, 433-437.

20 W. C. Koehler, Magnetic structures of rare earth metals and alloys, in Magnetic properties of rare earth metals, Springer, US, 1972, pp. 81-128.

21 S. B. Rochal, O. V. Konevtsova, A. E. Myasnikova and V. L. Lorman, Nanoscale, 2016, 8, 16976-16988.

22 D. L. Caspar, Biophys. J., 1980, 32, 103-138.

23 M. Brackmann, S. Nazarov, J. Wang and M. Basler, Trends Cell Biol., 2017, 27, 623-632.

24 J. Rubinstein, J. Math. Phys., 1970, 11, 258-266.

25 A. Maghsood, A. Chatterjee, I. Andricioaei and N. C. Perkins, Biophys. J., 2017, 113, 195-205.

26 N. J. Shikuma, M. Pilhofer, G. L. Weiss, M. G. Hadfield, G. J. Jensen and D. K. Newman, Science, 2014, 343, 529533. 